\title{
Privatización (de la administración) Privatization (of the administration)
}

\author{
Lucylea Gonçalves França \\ Universidad Federal do Maranhão \\ lucyfranca03@hotmail.com
}

Recibido / received: 27/11/2016

Aceptado / accepted: 02/03/2017

\section{DOI: https://doi.org/10.20318/eunomia.2017.3822}

\begin{abstract}
Resumen
Este artículo examina los recientes cambios en la actividad administrativa de los países cuyos mercados de servicios públicos han sido liberalizados en el nuevo marco de influencia neoliberal. Con la aparición de funciones públicas asociadas a la creciente extensión de las actividades económicas, los nuevos reglamentos se evaden de las exigencias tradicionales del régimen jurídico administrativo. La evolución del Estado social ha impulsado, también, una actividad administrativa global dirigida hacia los mercados liberalizados, que es responsable de difundirlos en nuevos entornos tras los procesos de privatización de servicios de interés general. En ese sentido, esa uniformización de normativas del Estado administrativo inicia la aplicación de métodos desarrollados para permitir el ejercicio eficaz de las prestaciones sociales y el derecho al acceso a la información al usuario. ¿Es posible y eficaz esa unificación para la mejora de los servicios de interés general? Esas prestaciones obtienen el reconocimiento de la responsabilidad estatal compleja en la medida en que exige averiguar el nivel de compromiso con el ciudadano, usuario de las prestaciones sociales. Ese análisis muestra la importancia de indagar cómo actúa el Estado de Brasil a la hora de evaluar los diferentes instrumentos jurídicos y materiales de las nueva administración pública global.
\end{abstract}

\section{Palabras clave}

Derechos sociales, Regulación de servicios privatizados, Control Público.

\begin{abstract}
This article analyses the recent changes in the administrative activity of countries whose public service markets have been liberalized under the new neoliberal framework. With the emergence of public functions associated with the increasing extension of economic activities, the new regulations evade the traditional requirements of the administrative legal regime. The evolution of the social state has also promoted a global administrative activity directed towards the liberalized markets, which is responsible for diffusing them into new environments after the processes of privatization of services of general interest. In this sense, this standardization of administrative state regulations starts the application of methods developed to allow the effective exercise of social benefits and the users' right to access to information. Is it that unification possible and effective so as to improve the services of general economic interest? These benefits obtain the recognition of the complex state responsibility as far as it demands to ascertain the level of commitment to the citizens, who are users of the social benefits. This analysis shows the importance of investigating how the Republic of Brazil acts to assess the different legal and material instruments of the new global public administrations.
\end{abstract}

\section{Keywords}

Social rights, Regulation of privatized service, Public Control. 
SUMARIO. 1. Introducción. 2. El contexto de los derechos sociales en la nueva administración pública operada en los mercados liberalizados: retos y contradicciones. 3 . ¿El control de los mercados liberalizados desde la perspectiva del Derecho administrativo global es posible? 4. A modo de conclusiones.

\section{Introducción}

Este trabajo pretende analizar de qué modo el nuevo modelo de Administración pública, dirigida a la regulación de los servicios de interés general, satisface y acompaña la consolidación del Estado de derecho y en qué medida muestra la nueva configuración de las prestaciones de los derechos sociales en el proceso progresivo de mercantilización de sectores económicos privatizados.

El incremento de las demandas sociales de los últimos cincuenta años ha marcado el rumbo del Estado occidental en su búsqueda de nuevas formas e instrumentos de intervención en la economía. La tendencia en el inicio del siglo XXI es de casi total desplazamiento de los sistemas jurídicos hacia la naturaleza privada de las prestaciones, siguiendo las condiciones fijadas por el modelo neoliberal y el capitalismo más desarrollado. Dentro de la disciplina jurídica, el Derecho Administrativo, las nuevas tecnologías y la expansión de la economía global juegan un papel fundamental hacia una gobernanza global.

En muchos países, ese influjo estuvo asociado a la reforma de la legislación administrativa de las nuevas constituciones democráticas de finales de los años 80 . La dogmática de los derechos fundamentales introducida en los sistemas constitucionales, incluyendo Brasil, ha tenido como consecuencia para la Administración pública la asunción de cambios de ordenación, condicionando incluso una nueva constitucionalización ${ }^{1}$ de los servicios públicos, concretizando el proyecto liberal en la liberalización de los mercados de estos servicios.

La separación clásica entre el Estado, el mercado y la sociedad ya no satisfacía a los intereses neoliberales y del capitalismo global de principios del siglo $X X I$, lo que supuso el inicio de la pérdida del protagonismo de las empresas públicas ${ }^{2}$ como elemento esencial de vinculación entre el interés estatal y las prestaciones sociales. El Estado empresario deja de ser hegemónico, es decir, de estar fuera de los mercados y tener privilegios orientados al monopolio, lo que ha dado paso a nuevos instrumentos de intervención que ya no encajan en la estructura de la disciplina jurídica más tradicional del derecho administrativo.

En los sectores de la economía en que intervenían, los órganos y empresas estatales gestoras de servicios públicos han tenido históricamente reconocidas posiciones de privilegio. El nuevo régimen administrativo aplicable a los mercados liberalizados no significó una progresiva evolución de los derechos sociales, ajustándose técnicamente a los nuevos patrones de la economía de mercado.

\footnotetext{
${ }^{1}$ Las reformas constitucionales en Brasil, tras los cambios de los artículos 173 y 174 de la Constitución Federal de 1988, crearon las bases para el desarrollo del proceso de privatización de los sectores de energía y telecomunicaciones, con la liberalización de servicios públicos antes operados por empresas públicas que tenían monopolios estatales. En ese sentido, el neoliberalismo fue asimilado por un sistema Constitucional adaptado a ese nuevo modelo a través de cambios constitucionales realizados a partir de 1998, en el gobierno del Presidente Fernando Henrique Cardoso.

2 Sobre la actuación de las empresas públicas en el modelo económico de los Estados democráticos de derecho, véase Martín-Retortillo (1991) y Muñoz Machado (1998).
} 
Aunque el modelo haya sido básicamente el norteamericano, hay notoria influencia europea y del "welfare estate" en ciertos sistemas que pasaran a ofrecer más resistencia al pensamiento administrativo y constitucional que otros. La especialización de la función reguladora dependió de la reacción del liderazgo más liberal en los distintos países latinoamericanos y fue ordenada de modo poco sistemático en la mayoría de ellos. El papel que juega cada país ha sido distinto, generando efectos secundarios de descontrol en la conducta administrativa y en el uso de los instrumentos normativos y ejecutivos de regulación.

En Brasil, la experiencia vigente acompañó a la tradición de la doctrina de los servicios públicos, por lo que sigue teniendo importancia crucial en la explicación de las decisiones económicas y sociales desde el modelo de Administración pública y desde la perspectiva de la libertad empresarial. Allí, la ausencia de mecanismos de regulación eficientes dificultó que operase la plena competencia, luego la noción de control del mercado es casi estática y estereotipada.

Los gobiernos, desde el inicio del proceso de privatización hasta hoy y más allá de las diferencias estructurales existentes, no están abiertos a romper posiciones económicas privilegiadas y se resisten a sustituir los monopolios de infraestructuras por la aparición de la plena competencia. En ese sentido, Brasil persiguió el modelo de regulación tradicional pero impuesta desde otros modelos distintos, dirigidos a la exclusividad, la preservación del monopolio y al no control en la mayoría de los servicios liberalizados. Ese ambiente se traslada al regulador, una figura estatal sin prestigio y con poca eficiencia, que no se adecua a la lógica de la libre competencia, sino a las garantías de los intereses y privilegios políticos, con reservas de prerrogativas que fomentan, además, la consolidación de los órganos de regulación dependientes y condicionados por los sectores que supervisan.

Esa conjunción anormal también contradice la visión de que existe una regulación linear y homogénea en los países con la misma línea de nueva regulación. El mantenimiento de ciertas reservas marcó de modo definitivo ese proceso marcado por las imprecisiones regulatorias y la continuidad de imposiciones de algunos privilegios estatales, antes destinados a las empresas públicas y ahora a la consolidación de un proceso económico que todavía no alcanzó la privatización y liberalización completa de los mercados.

2. El contexto de los derechos sociales en la nueva administración pública operada en los mercados liberalizados: retos y contradicciones

Los derechos sociales, como acciones que nacen de las necesidades de los individuos, pasan a estar vinculados a los mercados y operan dentro del marco de la incapacidad de la persona para la obtención de bienes o servicios necesarios para su existencia. Esta es la posición de Lema (2010, p. 181)3, que afirma que la universalidad de los derechos sociales debe ir más allá del conjunto de las prestaciones dirigidas hacia los que, de hecho, las necesitan.

Las perspectivas de las administraciones públicas han cambiado; se mueven en la gran encrucijada posmoderna, es decir, es más adecuado afirmar que siguen con el fin de hacer frente a la siempre creciente extensión y complejidad de los mercados y la casi total incapacidad pública para mantener, de modo eficaz, las tareas administrativas históricas del modelo de Estado social tradicional.

\footnotetext{
${ }^{3}$ En ese sentido, para Kotzur, la universalidad no es en sí estática, sino un proceso continuado y progresivo (2012) p. 229.
} 
La mercantilización de los servicios públicos se alinea con el discurso del permanente estado de crisis fiscal del Estado social para justificar las políticas neoliberales. Además, está presente la influencia de los grupos de presión dentro del Estado, incluyendo el freno de las políticas de control y regulación, así como el uso de cambios legislativos y constitucionales. En ese contexto, los sistemas jurídicos dibujaron un perfil más privado y desregulado, dirigido a las empresas globales que operan en los mercados de los servicios sociales.

En resumen, son los intereses corporativos, las crisis económicas y el endeudamiento público, los límites reales al discurso de rechazo al Estado de regulación, por lo tanto, existe la huida de muchos países de las políticas de bienestar social.

Las reformas más relevantes en el Derecho Administrativo del siglo XXI refuerzan la vis a vis del contenido privado de ciertas actividades estatales y de los servicios públicos más típicos. En ese largo recorrido, las constituciones han mantenido el núcleo duro de las prestaciones bajo la protección de los derechos sociales, pese a que se ha abandonado la planificación por parte de las Administraciones Públicas abandonaran la planificación, dando paso a la emergencia de un perfil de liberalización y de regulación, a veces, muy poco eficiente.

En ese sentido, independientemente del éxito regulatorio real, la función estatal tendría que ocupar un papel secundario, coadyuvante y equidistante de los mercados pero todavía imprescindible, porque compete a las administraciones públicas ser responsable por mantener la sobrevivencia de los derechos sociales. Ampararse bajo la influencia de los mercados sin estar dirigido por él es el objetivo del nuevo régimen jurídico, aunque pocos sistemas han logrado consolidar las bases de esas ordenaciones revitalizadas. Desde luego en Brasil, esa es la vuelta de tuerca que se espera en las bases del régimen de derecho público, aun no logrado en nuestros días.

Sin embargo, tampoco han cambiado tanto los objetivos de la Administración pública en su historia más reciente. Mientras la función regulatoria, normativa y ejecutiva del Estado surgió de la incorporación de empresas privadas al servicio del desarrollo de las actividades de utilidad pública a finales del siglo $\mathrm{XX}$, tales fenómenos, todavía se asocian al mismo modelo de legitimación administrativa tradicional defendida desde Duguit $(2007)^{4}$, es decir, entre los siglos XIX y XX, cuando los organismos y empresas gestoras de actividades económicas estaban reconocidos y gozaban de una posición privilegiada en los mismos sectores que regulan.

La doctrina jurídica sigue centrada en los puntos fundamentales del antes y ahora en el régimen jurídico de muchos países, aunque especializado dentro de la función reguladora que unifica el modelo de Estado administrador, pero con nuevos retos normativos. Por lo tanto, ese es, para Harlow (2006, p. 189), el llamado pluralismo legal global, donde se incluye las reglas del juego, el mismo juego y los jugadores.

Los países persiguen los desafíos históricos pero dentro de las contradicciones internas, basados en necesidad de concebir un modelo de administración con capacidad de imponerse en la defensa de la universalidad de los

\footnotetext{
${ }^{4}$ Además, para Wahl, las organizaciones constitucionales y la evolución del Estado social han permitido decir del Estado actual es sobretodo "Estado Administrativo" (2013).
} 
derechos sociales, necesitando para eso preservar su potestad administrativa eficiente e independiente frente a los mercados.

Por otro lado, las prestaciones materiales de los servidos de interés general supondrían tener los mercados bajo el control de la plena competencia, es decir, la personificación del modelo ideal de los servicios públicos económicos, liberalizados y controlados. Partiendo de una situación fáctica, el conjunto de la administración se ordenó en el Estado social como lo que es, por su contenido, más allá de definirse en el modelo liberal, fue más importante obligarse por lo que hace (1997, p. 35).

Sin embargo, en la ejecución del interés general, la Administración burocrática y centralista no ha podido legitimarse con base en la normativa en vigor, muchas veces subrayada y construida en el empeño de la realización de las prestaciones materiales directamente por el Estado. En Brasil los cambios legislativos han permitido el canje en el sistema interventor. Además, el camino del Estado proveedor ya no es el buscado por el Estado de principios del siglo XXI.

Mientras tanto, hablando de regulación es importante matizar que ya en los años 50 la escuela de Chicago advertía de la necesidad de una especie de regulación asistida para empresas situadas fuera del mercado (sobre el modelo tradicional y la evolución histórica de la regulación, véase Stigler, 1971, pp. 3-21), es decir, a la vez de la formación de los monopolios naturales que impedirían el acceso a las infraestructuras de otros reguladores, era evidente el peligro de un impedimento errante a las nuevas inversiones, eficiencia, innovaciones y el desarrollo. Se habla de la necesidad de fijar parámetros de un derecho administrativo global ${ }^{5}$, en una especie de lucha por domesticar la globalización ${ }^{6}$, aunque el mayor desafío consiste en fijar contenidos ideológicos internos y hegemónicos, que robustezcan los estereotipos de la regulación que, hoy por hoy, se presenta con fallos en los instrumentos jurídicos y ejecutivos en diversos países tanto de Europa como de América. Es por lo tanto, la eterna paradoja descrita por Kelsen (2003, pp. 69-70), cuando dice que goza de un favor entre los juristas presentar como imposible aquello que en realidad solo es políticamente indeseado porque se opone a ciertos intereses particulares.

En concreto, la contradicción del régimen de los mercados liberalizados de los servicios de interés general se refuerza por la construcción de una función reguladora emergente pero poco realista, dando paso a un control poco efectivo y al servicio de las relaciones concretas del Estado y del mercado en cada país. Los sistemas jurídicos que no acompañan las circunstancias administrativas y políticas universales sufren influencias internas muy distintas entre uno y otros. De modo concreto, países que optaron por privatizar y regular sus servicios públicos como Brasil se encontraron el problema de la consolidación del ambiente regulador, con agencias y entidades independientes fuera del mercado que no existían hasta entonces en el modelo administrativo brasileño y que pasarían a operar de manera ineficiente, por no adecuarse de modo definitivo al seno de los sectores que deberían supervisar.

3. ¿El control de los mercados liberalizados desde la perspectiva del Derecho administrativo global es posible?

Es evidente que sin los apoyos de los organismos y las influencias al servicio del capitalismo global no se puede entender los cambios del Derecho administrativo hacia el ambiente regulador, pero también es cierto que ya se ha superado las expectativas

\footnotetext{
5 En el contexto de la relación individuo-Estado y participación ciudadana en la teoría política, destacamos Sánchez Morón (1980, p. 285).

${ }^{6}$ En la definición semántica de gobernanza global, véase Kingsbury y Steward (2016).
} 
en establecer paralelismos evidentes de aproximación entre el modelo europeo y brasileño de los mercados de servicios de interés general liberalizados. Ni siquiera por su nomenclatura se supone un acuerdo, es decir, mientras que en Europa se fortaleció dentro del derecho administrativo el contenido de los servicios de interés social, en gran parte de América se sigue con la traducción de estos como servicios públicos ${ }^{7}$.

En esas mismas instancias se puede encajar una posible excepcionalidad de las prestaciones sociales, donde los derechos sociales no se han concretado ni antes ni después de las privatizaciones. Es decir, mientras el mercado liberalizado no acaba de consolidarse, sigue mermado por reservas de servicios económicos a las administraciones públicas, en regímenes de privilegios y resistencia al control público por parte de las empresas privadas que actúan en los sectores privatizados.

Es un punto de inflexión que los servicios públicos deben superar en la actualidad, a riesgo de que no lleguen a ser operados con eficiencia, desde una perspectiva indisoluble del control a partir de la regulación. El sistema jurídico debe ir más allá de garantizar la prestación de las necesidades básicas, es decir, dar paso a una acción positiva capaz de consolidar derechos sociales económicos todavía no alcanzados por esquemas idénticos, sean antes o después de las privatizaciones.

La eficacia de las prestaciones sociales está en la universalidad formal, presente e ineludible desde el punto de vista de las necesidades previstas por instrumentos de control que aseguren el Estado democrático de Derecho. Sin embargo, ciertos mercados han sufrido más resistencia al control. Asimismo, muchos sistemas jurídicos han tenido más debilidad que otros a la hora de regular e imponer medios de control. El sector eléctrico es un ejemplo de mercado que en la mayoría de los países sigue manteniendo posiciones de privilegio en Europa y Latinoamérica.

De hecho, la existencia de mercados en plena competencia se presenta, después de casi veinte años de liberalización, como una expectativa no cumplida. Ese equivoco regulatorio existe justificado por ciertos ambientes normativos y políticos que rechazan cualquier forma de control, por lo tanto, asumen una regulación casi ficticia. Es decir, de nuevo la regulación, como modelo de intervención al modo anglosajón, es inalcanzable en los países de tradición europea, como $\mathrm{Brasil}^{8}$, con realidades jurídicas, políticas y administrativas muy distintas y que quizás por eso no consiguen asimilar y enlazar un adecuado parámetro que justifique la adaptación aun derecho administrativo global, generando una clara visión equivocada e imprecisa de regulación.

Regulación es movimiento y proceso, tampoco los mercados son estáticos y herméticos, es inapropiado dejar de atribuir a los organismos administrativos, también mutantes, la competencia y responsabilidad de generar el marco sostenible de protección a las prestaciones sociales. Asimismo, es difícil de creer que la regulación con eficiencia pueda operar sin control y fuera del marco de la competencia plena, como dice Muñoz Machado (2009, pp. 18-20).

\footnotetext{
${ }^{7}$ Lo que ha sido traducido por Villar Ezcurra y Villar Palasi (1980, p. 14) como una categoría jurídica descategorizada, un ente ubicuo, que cada uno interpreta a su manera por estar definido por distintas formas, que siempre estuvo centrado en el control como un requisito vital de la potestad administrativa.

8 Sobre el tema del régimen jurídico de las administraciones publicas de Brasil hacemos especial referencia a Moreira Neto, que destaca la llamada administración independiente tradicional del Derecho anglosajón, que llamo la atención y pasó a ser adoptada por muchos países de Europa y América, en la que demuestra una peculiar eficiencia operativa en países política y económicamente abiertos, en razón de especialización, flexibilidad, independencia, celeridad, proximidad y apertura social de su acción (2000, pp. 79-80).
} 
Por otro lado, la función estratégica, económica y política de las empresas públicas ha tenido su importancia y sigue estando muy presentes en Brasil. Detrás de este fenómeno están aspectos políticos y de financiación del Estado, que merman la relevancia del control en esas prestaciones complejas en su contenido, realizadas directamente por el Estado o no.

Las dimensiones de las relaciones entre Estado, sociedad y mercado parecen confundir las implicaciones de las administraciones públicas generando un un profundo abismo en la "independencia condicionada" y su subordinación al "enmarcamiento" (Kliksberg, 1984, pp. 23-42) de las instituciones. Tras una copiosa doctrina, trato de investigar sobre la redefinición del contexto de actuación de las nuevas figuras e instituciones jurídicas en el ambiente pos-regulador. En resumen, de un lado hay el régimen normativo garante, condicionado a la tradición histórica del derecho administrativo de cada país, y de otro, el derecho administrativo de la regulación, impuesto por una cultura global y generando, como dice Wahl (2013, pp. 133-134, a propósito de la experiencia alemana), el desglose del concepto de responsabilidad estatal.

Profundizar en la teoría de los conceptos clave del derecho administrativo puede que permita el reencuentro de la esencia del derecho público, quizá, las administraciones encuentren el camino hacia la better regulation, arropando las dificultades de eliminar y sustituir las prácticas contrarias a la modernización de las funciones administrativas y dando paso a una real tarea renovadora.

Así pues, de un lado quedan los roles de los mercados capitalistas occidentales, que dan mayor preeminencia a la libertades de los empresarios, y de otro un injusto desplazamiento de las políticas hacía la reducción de los derechos sociales, bajo el argumento de que muchos de los destinatarios de esas prestaciones las reciben parasitariamente, aunque esa no sea la realidad.

Las capacidades económicas de los ciudadanos provocan que sólo aquellos con medios económicos puedan sostener el Estado social, mientras tanto, lo que parece ser ilusorio pues necesita de un sistema impositivo justo para reducir las desigualdades y garantizar la universalidad de las prestaciones.

Los instrumentos administrativos poco eficientes, burocráticos y politizados son el contexto que siembra la desigualdad y la injusticia social, al restringir o impedir el alcance de los derechos sociales.

Todavía hay otras variantes. La eficacia de las prestaciones tienen más que ver con la forma con que los servicios son realizados. En un sistema económico justo la eficacia fiscal acompaña la cobertura y reduce los fraudes de modo preventivo y represivo. La supuesta incapacidad de los países para hacer frente a la crisis del estado de bienestar y a cualquier dificultad financiera enfrentada por los países capitalistas es, en general, un discurso operado desde dentro de los mercados y que hace hincapié en la retórica neoliberal para promocionar más recortes sociales.

En el caso de Brasil, la dirección de las políticas en manos de gobiernos más conservadores y contrarios al modelo tradicional de regulación ha generado acciones opuestas a la lógica del control. Por ejemplo, los mercados privatizados, como de las telecomunicaciones y el sector eléctrico, se mantuvieron cerrados como mercados monopolizados y los concesionarios de redes de infraestructuras han podido prestar en régimen de exclusividad los servicios que les adjudicaron los modelos de privatización originarios, estando permanentemente vetadas la aparición de operadoras entrantes. 
La aparición de esa nueva (re)designación de intervención motivó un cambio del modus operandi de las estructuras administrativas. La economía de los medios y la crecente extensión de servicios privatizados exigió nuevas reglas de derecho administrativo. Sin embargo, la regulación de instituciones privadas, mucho más allá de las administraciones públicas tradicionales, exige que el llamado Estado regulador pueda operarse desde el punto de vista de las leyes de control, hacia la transparencia real de la prestación de estos servicios, ese, además, es el freno de la dimensión pública y administrativa de los derechos sociales en el proceso de desmercantilización.

La acción administrativa, por lo tanto, aunque elemento limitativo de las libertades individuales, tiene en la regulación formal su función estatal preponderante. Es posible que la cultura de la legalidad de las democracias posmodernas promuevan y condicionen los cambios constitucionales con la excusa de imponer el reduccionismo de los derechos sociales.

Esa también ha sido una lucha insana en el gran paradigma actual, exigiéndose sobre todo a la administración, en sus múltiples campos de actividad, que dejase de producirse en la simplicidad (Weil, 1989, p. 66), es decir, que los mercados privatizados sean atendidos de modo eficiente. En ese contexto, es necesaria la sistematización de funciones normativas, ajustándolas distintas formas y variables flexibles, sólo posible con la modernización de regímenes aplicables y operados desde la eficiencia. Así, los auténticos problemas se sitúan en tres dimensiones: la complejidad de los regímenes administrativos vigentes, las prestaciones que ya no están en la esfera de las acciones estatales y la dialéctica caótica de la disociación entre los métodos regulatorios, es decir, la distancia entre los fines y los medios de la actividad regulatoria de los entes administrativos.

Se destaca la accountability de tradición anglosajona, que propone superar el modelo tradicional de control del gasto público, para alinearse mucho más a la transparencia y la intervención general buscada por los países latinoamericanos, donde se tengan en cuenta aspectos como el político, el judicial, el contable y el financiero, además de la calidad. Una reforma administrativa que no solamente busque la reducción del déficit público y los resultados antes que el bienestar social, es más probable que garantice la eficacia de los instrumentos de participación ciudadana, el consenso social y la acción de las administraciones públicas en defensa de los derechos sociales.

Los privilegios de las administraciones, muy presentes en el modelo tradicional, han significado el estado de ausencia del control administrativo o su contenido innecesario frente a derechos fundamentales poco consolidados. Solo la existencia de cierto control judicial, vertical y transcendente conlleva, en estos momentos, un sistema de conexión entre el pueblo y el gobierno. Sin embargo, el razonamiento basado tanto en criterios extrajudiciales como económicos, políticos o éticos también redefine el perfil de las políticas de composición de los intereses generales desde reformas constitucionales. Por lo tanto, sería muy amplio el abanico de influencias para la politización de los métodos de regulación, llegando incluso a lograr métodos organizativos y procedimentales distintos, capaces de romper con la cultura de la función administrativa clásica, formal y constitucional existente.

Es arriesgado vincular los métodos de control a la discrecionalidad administrativa, dentro de una especie de sumisión precaria de las prestaciones sociales a las instancias ocupadas por gestores políticos que, en regla, cambian de posición o de discurso cuando cambian de partido político, o cuando alcanzan la jefatura del ejecutivo. 
En ese proceso de avance y retroceso, el reto de la globalización jurídica administrativa se ubica en conceptos-principios lógicos fundamentales, es decir, el derecho a la buena administración, regularidad procedimental, participación ciudadana, transparencia y buena fe, donde los intereses colectivos y difusos envueltos de manera reglamentaria puedan ser tomados en consideración con gran protagonismo (Nieto, 1975, pp. 26-27). Lo que conlleva afirmar, además, de modo más preciso, que el derecho administrativo, dentro de los estándares de la dogmática tradicional, bien ordenada y construida a partir de la posguerra, ha perdido lugar de modo definitivo (Schmidt-Aßmann, 2006, p. 31).

Sin embargo, mejorar el control de la administración y el aparato estatal juega un papel fundamental en el derecho administrativo global. Mientras tanto, su objeto central ya no es la propia administración pública, puesto que ya no está vigente una estructura piramidal impositiva, estática, cerrada y auto determinada. Las demandas por el protagonismo de la atención ciudadana y la transparencia en las acciones de interés general son tan importantes como el control mismo a las actividades privadas que ejecutan los servicios sociales. Por lo tanto, ese ambiente ya no es homogéneo y exige normas jurídicas específicas dentro del régimen de las administraciones públicas. Sin embargo, se constata que ya no se involucran al esquema tradicional, basado en la discrecionalidad de la conveniencia pública, con estándares inmutables en sus formas de administrar.

Desde el punto de vista de la cooperación residual de la sociedad frente a las administraciones públicas es evidente que la acción estatal de hoy se renueva hacia relaciones más amplias, directas y transparentes. La esfera social de la administración nacional, aislada, solemne, casi intocable, exige abrir el paso a nuevos conceptos claves como la gobernanza y transparencia.

Estos dos lados de la Administración pública, el antes y el después, al fin y al cabo son un mismo sentir, el rescate del núcleo duro de las normas de derecho público de hoy y de siempre. No se trata, por lo tanto, de una administración que se opere en dos velocidades. Allí está ubicado el desafío simbólico que difunde el Derecho administrativo global, es decir, incorporar más mecanismos que refuercen la legitimidad democrática para garantizar la permeabilidad necesaria al tejido del Estado y de la administración dentro de los mercados. Esa versatilidad no es fácil de alcanzar, pero tiende a imponerse, hoy por hoy, como estrella y motor conducente a un desarrollo preciso de procesos eficientes, legales y proporcionales de las necesidades sociales.

Es inevitable reconocer que en ese transcurso histórico hay una progresiva pérdida por parte de la ciencia jurídico-administrativa de su papel orientador, o por lo menos el sentimiento de cierta amenaza, provocado por la incertidumbre en una adaptación que puede ser determinante para decidir acerca de las estrategias o resultados de estos servicios. Las actividades de interés social llevan a los países a asumir ese riesgo regulatorio, definido por Ortiz (2006, p. 27) como la imprevisibilidad e inseguridad además de la politización de las decisiones administrativas.

\section{A modo de conclusiones}

El peligro regulatorio es real cuando existe la mala gestión de los procedimientos. EI caos acompaña la configuración distorsionada del Derecho administrativo global y de las normas de derecho público. La ya plural y compleja relación entre la economía y los derechos sociales se debilita por el abandono del equilibrio entre poderes y contrapoderes del Estado y de la sociedad. En la fuerza del Estado Administrativo también se ubica su debilidad, en el riesgo de la ineficacia de los instrumentos de 
regulación está él éxito de la actuación de los agentes administrativos. Sin embargo, hay conflictos reales en el desajuste del método de check and balance administrativo, es decir, la incautación de la administración pública y prácticas poco democráticas empeoran el escenario anterior. Así, dentro de estructuras de poder marcadas por otros factores diversos e intereses personales se crea el caldo de cultivo de prácticas muy nefastas como la corrupción, el clientelismo y/o exceso de centralismo de las Administraciones.

La fragmentación del Estado regulador y su desorden, por lo tanto, generan debilidad de los mecanismos de control y vulneran el equilibrio de las fuerzas que actúan dentro de las Administraciones públicas. En resumen, la ordenación de la actividad económica de interés general por instrumentos regulatorios se encuentra tutelada por el contexto político donde se ubican las administraciones. Por lo tanto, ocupa una posición estratégica de sobre posición de lo económico a lo institucional.

Los instrumentos norteamericanos de normativa administrativa puede que sirvan de parámetro y, de hecho, han llegado a afectar muchos países. Mientras tanto, el esquema del control y eficiencia es una realidad poco presente en la realidad jurídica de Brasil. También es cierta la existencia del fenómeno de la europeización del Derecho Administrativo, en ese proceso se va produciendo, a costa de avances y retrocesos, el derecho administrativo en los últimos años.

La captura de las Administraciones, es decir, la manipulación por parte de la política y del poder ejecutivo de los instrumentos de control y transparencia, influyen para imposibilitar una buena administración. En ese sentido, con recursos de transparencia de las acciones administrativas y accountability, el ciudadano, el administrado, el cliente, dejan de ser entes pasivos e inexpresivos en la relación con el Estado. Su fragilidad está en el control político más allá del jurídico. Sin embargo, los recursos públicos, deberían, como dice para Rodríguez-Arana (2010, p. 73), estipular como regla la transparencia cuando sea posible y tanta restricción como sea necesaria.

Está claro que en el seno del derecho administrativo internacional los agentes públicos y privados que ejercen funciones públicas de interés general, se someten al control por parte de los órganos y/o instancias públicas competentes. En ese proceso, lo establecido directamente por la Constitución y las leyes de contenido específico guarda un valor esencial, cuyos límites se asocian a los derechos fundamentales de protección del interés público, la seguridad nacional, la protección de datos y las relaciones internacionales.

El resultado en la búsqueda de un derecho administrativo global está en la confluencia de un sistema regulatorio transnacional y la elaboración de mecanismos adaptados a las realidades locales, pero más rígidos y capaces de promocionar la cooperación hacia la regulación desde los principios clave universales, dentro del sistema de buen gobierno, y en contra la evasión de divisas fruto de la corrupción. Un sistema fluido y efectivo, que permita hablar de un derecho administrativo basado en sistemas jurídicos internos plenamente adaptados a las realidades internas y asemejados a experiencias de éxito en otros países, y que pueda señalar mecanismos más eficientes rumbo a la real protección de los derechos sociales.

\section{Bibliografía}

ARIÑO ORTIZ, G. (2006), "Fallos y logros de los sistemas regulatorios”, Regulación, desregulación, liberalización y competencia, pp. 23-40.

BAÑóN, R. Y CARRILLO, R. (1997), La nueva administración pública, Alianza 
Universal, Madrid.

DUGUIT, L. (2007), Las transformaciones del derecho público y privado, Comares, Madrid.

FIGUEREIDO MOREIRA NETO, D. (2000), "Consideraciones sobre la participación en el Derecho Comparado Brasil-España", Revista de Administración Pública, n¹52, pp.73-91.

HARLOW, C. (2006), "Global Adminisrative Law: the Quest of principles and values", The European Journal of Internacional Law, Vol. 17, $\mathrm{n}^{\circ}$ 1, pp. 187-214.

KELSEN, H. (2003), La paz por medio del Derecho, Trotta, Madrid.

KINGSBURY, B. y STEWARD, R. (2016), Hacia el derecho administrativo global: Fundamento, Principios y ámbito de aplicación, Global Law Press e Instituto de Administración Pública, Sevilla - Madrid.

KLIKSBERG, B. (1984), "Propuesta de un modelo metodológico para la investigación sistemática del complejo de empresas públicas en países de américa latina. Modelos y experiencias latinoamericanos". En: KLIKSBERG, B. y SULBRANDT, J. (comp.), Para investigar la Administración Pública: Modelos y experiencias latinoamericanas, Alcalá de Henares, Instituto Nacional de Administración Pública, p. 23- 42.

KOTZUR, M. (2012), “Derechos Humanos y el Tribunal de los derechos humanos”, Anuario Iberoamericano de Justicia Constitucional, $\mathrm{n}^{\circ}$ 16, pp. 225-249.

LEMA AÑÓN, C. (2010),“Derechos sociales, ¿para quién? Sobre la universalidad de los derechos sociales", Derechos y libertades, n² 22, Época II, pp.179-203.

MARTíN-RETORTILLO, S. (1991), Derecho administrativo Económico. I, La Ley, Madrid.

MOREIRA NETO, D. (2000), "Consideraciones sobre la participación en el Derecho

Comparado Brasil-España”, Revista de Administración Pública, n¹52, pp. 73-91.

MUÑOZ MACHADO, S. (1998), Servicio Público y Mercado. IV. El Sector Eléctrico, Civitas, Madrid.

MUÑOZ MACHADO, S. (2009), Derecho de la Regulación Económica. I. Fundamentos e Instituciones de la Regulación, lustel, Madrid.

NIETO, A. (1975), "La vocación del derecho administrativo en nuestro tiempo", Revista de Administración pública, $\mathrm{n}^{\circ} 76$, pp. 9-30.

RODRÍGUEZ-ARANA MUÑOZ, X. R. (2010), "Derecho Administrativo Global: Un derecho principal", Revista Andaluza de Administración pública, n 76, pp. 15-68.

SANCHEZ MORÓN, M. (1980), "Introducción al régimen jurídico de la empresa pública en España", Revista de Administración Pública, nº 93, pp. 67-128.

SCHMIDT AßMANN, E. (2006), "Cuestiones fundamentales sobre la reforma de la Teoría General del Derecho Administrativo". En: BARNES, J. (ed.), Innovación y Reforma en El Derecho administrativo, Global Law Press e Instituto de Administración Pública, Sevilla - Madrid. pp. 21-140.

VILLAR EZCURRA, J. L. y VILLAR PALASI, J. L. (1980), Servicio Público y Técnicas de Conexión. Estudios de Administración Centro de Estudios Constitucionales, Madrid.

WEIL, P. (1989), Derecho Administrativo, Cuadernos Civitas, Madrid.

WAHL, R. (2013), Los últimos cincuenta años de Derecho administrativo alemán, Marcial Pons, Madrid. 